\title{
Higher levels of neurofilament light chain and total tau in CSF are associated with negative outcome after shunt surgery in patients with normal pressure hydrocephalus
}

\author{
Madelene Braun ${ }^{1}$, Caroline Bjurnemark ${ }^{2}$, Woosung Seo ${ }^{3}$, Eva Freyhult ${ }^{4}$, Dag Nyholm ${ }^{1}$, Valter Niemelä ${ }^{1}$,
}

Kaj Blennow ${ }^{5,6}$, Henrik Zetterberg $^{5,6,7,8,9}$, David Fällmar ${ }^{3}$, Kim Kultima² $^{2}$ and Johan Virhammar ${ }^{{ }^{*}}$ (D)

\begin{abstract}
Background: Lumbar punctures are a common examination in the work-up of patients with idiopathic normal pressure hydrocephalus (iNPH) and cerebrospinal fluid (CSF) biomarkers should therefore be available for use in selection of shunt candidates. The aim of this study was to investigate if CSF biomarkers are associated with outcome after shunt surgery alone or in combination with comorbidity and imaging markers, and investigate associations between CSF biomarkers and symptoms.

Methods: Preoperative CSF biomarkers were analyzed in 455 patients operated with shunt surgery for iNPH at a single center during 2011-2018. Symptoms before and 12 months after shunt surgery were graded with the Swedish iNPH scale. Neurofilament light chain protein (NfL), total tau (T-tau), phosphorylated tau (P-tau) and amyloid beta1-42 (Aß1-42) CSF levels were measured. Evans' index and disproportionately enlarged subarachnoid space hydrocephalus were measured on preoperative CT-scans. Preoperative evaluation and follow-up 12 months after shunt surgery were available in 376 patients.
\end{abstract}

Results: Higher levels of NfL and T-tau were associated with less improvement after shunt surgery $(\beta=-3.10$, $p=0.016$ and $\beta=-2.45, p=0.012$, respectively). Patients whose symptoms deteriorated after shunt surgery had higher preoperative levels of NfL (1250 ng/L [IQR:1020-2220] vs. 1020 [770-1649], p<0.001) and T-tau (221 ng/L [IQR: 159-346] vs. 190 [135-261], $p=0.0039$ ) than patients with postoperative improvement on the iNPH scale. Among the patients who improved $\geq 5$ levels on the iNPH scale (55\%), NfL was abnormal in 22\%, T-tau in 14\%, P-tau in 6\% and A $1-42$ in 45\%, compared with normal reference limits. The inclusion of CSF biomarkers, imaging markers and comorbidity in multivariate predictive Orthogonal Projections to Latent Structures (OPLS) models to did not improve predictability in outcome after shunt surgery.

Conclusions: Higher levels of T-tau and NfL were associated with a less favorable response to shunt surgery, suggesting a more active neurodegeneration in this group of patients. However, CSF levels of these biomarkers can be elevated also in patients who respond to shunt surgery. Thus, none of these CSF biomarkers, alone or used in combination, are suitable for excluding patients from surgery.

*Correspondence: johan.virhammar@neuro.uu.se

1 Department of Neuroscience, Neurology, Uppsala University, Akademiska sjukhuset, ing 85, 75185 Uppsala, Sweden

Full list of author information is available at the end of the article permits use, sharing, adaptation, distribution and reproduction in any medium or format, as long as you give appropriate credit to the original author(s) and the source, provide a link to the Creative Commons licence, and indicate if changes were made. The images or other third party material in this article are included in the article's Creative Commons licence, unless indicated otherwise in a credit line to the material. If material is not included in the article's Creative Commons licence and your intended use is not permitted by statutory regulation or exceeds the permitted use, you will need to obtain permission directly from the copyright holder. To view a copy of this licence, visit http://creativecommons.org/licenses/by/4.0/. The Creative Commons Public Domain Dedication waiver (http://creativeco mmons.org/publicdomain/zero/1.0/) applies to the data made available in this article, unless otherwise stated in a credit line to the data. 
Keywords: Normal pressure hydrocephalus, Tau, Neurofilament light chain, Amyloid beta, Biomarkers, Cerebrospinal fluid, Lumbar puncture

\section{Background}

Idiopathic normal pressure hydrocephalus (iNPH) is characterized by a progressing gait disturbance, cognitive impairment and urgency incontinence. The condition is most often diagnosed in patients older than 60 years and prevalence increases with age $[1,2]$. Implantation of a shunt system reduces symptoms in $60-80 \%$ of patients [3-5]. Various examinations, such as magnetic resonance imaging of the brain and lumbar infusion tests, are used to aid the selection of patients for shunt surgery. Tests that reliably predict a negative outcome after shunt surgery are lacking and there are no cerebrospinal fluid (CSF) biomarkers that can identify patients who would or would not benefit from shunt insertion [6].

CSF neurofilament light chain (NfL) is a marker of degeneration of myelinated axons and is increased in neurodegenerative, neuroinflammatory, traumatic and cerebrovascular disorders [7]. Greater impairment is associated with higher levels of NfL in both patients with iNPH and patients with secondary NPH [8] and a postoperative reduction in NfL correlates with clinical improvement after shunt surgery [8].

Studies investigating the predictive value of NfL, total tau (T-tau) and amyloid beta1-42 (A $\beta 1-42)$ in lumbar CSF have most commonly been small, single-center studies including a limited number of patients $[9,10]$.

It has been suggested that analysis of T-tau and $A \beta 1$ 42 can differentiate between Alzheimer's disease (AD) and $\mathrm{iNPH}$ and could therefore have a diagnostic value in the work-up of iNPH [11-14]. The predictive value of these biomarkers for shunt responsiveness has been tested in previous studies with diverging results [10, 15-17]. Tau is thought to reflect neuroaxonal degeneration and it has been suggested that patients with high $\mathrm{T}$-tau and phosphorylated tau (P-tau) and/or low A $\beta 1$ 42 (reflecting sequestration of the protein in the brain in amyloid plaques) would be poor surgical candidates, but there is a large variation in the literature describing outcomes in this group $[17,18]$.

Lumbar punctures are a common examination in the work-up of patients with iNPH and routine at many centers. Analyses of CSF can thus easily be made in this group of patients. The clinical value of CSF biomarkers in patients with iNPH is not clear, with diverging results in previous studies, which have often had small sample sizes. In the present study, we aimed to investigate the value of NfL, T-tau, P-tau and A $\beta 1-42$ to predict outcome after shunt surgery and the association between the biomarkers and clinical symptoms.

\section{Methods}

\section{Study design and patients}

This was a retrospective single-center study including patients with iNPH who were treated with implantation of a shunt system during January 2011 to August 2018. CSF samples were collected preoperatively and evaluations of clinical symptoms were performed at the time of CSF sampling and 3 and 12 months after shunt surgery. Outcome after surgery in the primary statistical analyses was decided at 12 months after shunt surgery but results from 3 months after shunt surgery are included in the Supplementary results. Median time between CSF sampling and shunt surgery was 6 months [IQR 4-8] and between shunt surgery and 12 months follow-up it was 12 months [IQR 12-13].

\section{Inclusion and exclusion criteria}

Included patients met the diagnostic criteria of iNPH according to international guidelines [19]. Inclusion criteria were at least one analyzed CSF marker (NfL, T-tau, P-tau or Aß1-42) preoperatively and evaluation of symptoms before surgery with at least one domain from the iNPH scale or cognitive assessment with Mini Mental State Examination (MMSE). For analyses that included outcome after shunting, only patients with documented evaluation of symptoms preoperatively and at follow-up 12 months after shunt surgery (with at least one domain from the iNPH scale) were included. Other reasons for exclusion from analyses of outcome were follow-up at other hospitals, death before follow-up or any concomitant disease that made evaluations of outcome difficult, Fig. 1.

\section{Clinical evaluations}

All patients were examined by a specialized team including neurologists, neurosurgeons, nurses, physiotherapists and occupational therapists. The workup included CSF tap tests and lumbar infusion tests. Symptoms were graded using the MMSE and the iNPH scale [20]. The iNPH scale is based on grading of four major symptom domains (gait, balance, cognitive function and continence) and is used to assess a patient's disability. Each domain is scored between 0 and 100, where 0 represents the most severe state possible and 100 is the performance of an age-matched 


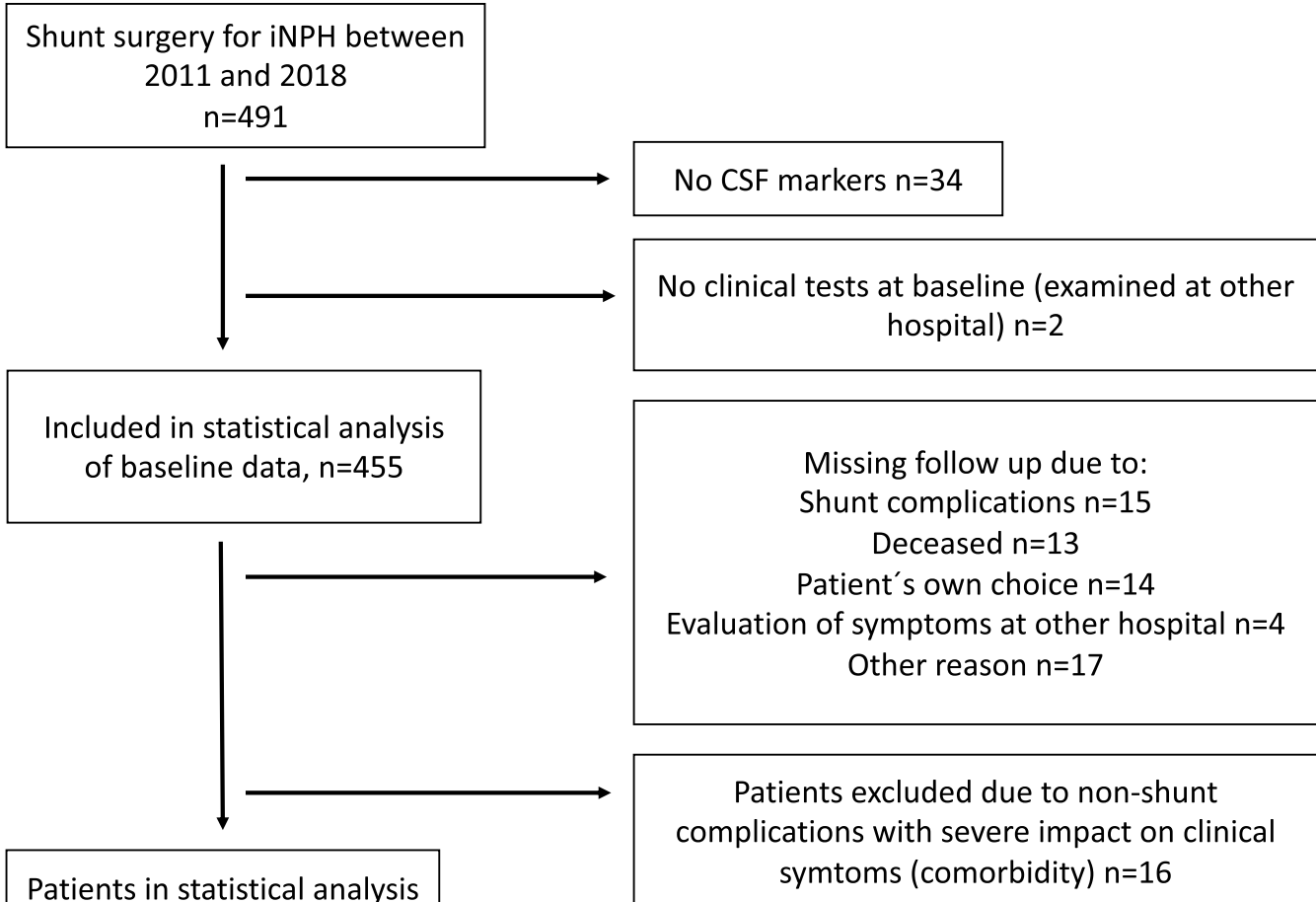

Fig. 1 Flowchart of patients included and excluded. A total of 376 patients had pre- and postoperative evaluations and were included in the statistical analysis of outcome

healthy individual. A total score is calculated as the average of the four domains (gait has double weight), or as the average of the available domains if any domain is missing. A subgroup of 162 (36\%) patients was examined with an alternative version of the Stroop color test and Stroop interference including only 24 colored circles/words instead of 100 squares as in the original iNPH scale. For these patients, the cognitive domain score was calculated using a modified conversion table (Additional file 1: Table S1). Outcome after shunting was calculated as the difference in iNPH scale score between 12 months follow-up post-surgery and the preoperative visit, referred to as delta iNPH scale. Outcome at 3 months after shunt surgery was included in the supplement.

Information regarding the following comorbidities was documented: history of ischemic stroke or transient ischemic attack, coronary heart disease defined as previous acute coronary syndrome, diabetes mellitus, oral anticoagulants and hypertension. Hypertension was recorded if the patient was on medication for hypertension, Table 1.

\section{Laboratory analyses}

Levels of NfL, T-tau, P-tau and A $\beta 1-42$ were analyzed from preoperative CSF samples. Samples were collected into polypropylene tubes and the first $3 \mathrm{~mL}$ of the lumbar tap were used for analyses. Samples were analyzed at the Clinical Neurochemistry Laboratory at Sahlgrenska University Hospital and laboratory technicians were blinded to clinical data. NfL level was measured using a commercially available enzyme-linked immunosorbent assay, in accordance with instructions from the manufacturer (UmanDiagnostics, Umeå, Sweden). T-tau, P-tau and A $\beta 1-42$ levels were measured using INNOTEST enzyme-linked immunosorbent assays in accordance with instructions from the manufacturer (Fujirebio, Ghent, Belgium). Procedures for acquiring high longitudinal stability in the measurements has been described previously and the coefficients of variation for the quality-control samples from the same lab was in a previous study between 7.1 and $11.5 \%$ for T-tau, P-tau, and A $\beta 1-42$ [21].

Laboratory-specific normal reference ranges: NfL: 40-60 years, $<890 \mathrm{ng} / \mathrm{L},>60$ years, $<1850 \mathrm{ng} / \mathrm{L}[22,23]$; T-tau: $<350 \mathrm{ng} / \mathrm{L} ;$ P-tau: $<60 \mathrm{ng} / \mathrm{L} ; \mathrm{A} \beta 1-42:>530 \mathrm{ng} / \mathrm{L}$ [23]. 
Table 1 Demographical data and preoperative symptoms

\begin{tabular}{|c|c|c|}
\hline & $\mathbf{n}$ & \\
\hline Age, years (range) & 455 & $75(50-89)$ \\
\hline Sex, male (\%) & 455 & $257(57)$ \\
\hline $\begin{array}{l}\text { Time from examination to shunt, months, median } \\
\text { (IQR) }\end{array}$ & 455 & $6(4-8)$ \\
\hline $\begin{array}{l}\text { Time from operation to follow-up, months, } \\
\text { median (IQR) }\end{array}$ & 376 & $12(12-13)$ \\
\hline \multicolumn{3}{|l|}{ Comorbidities } \\
\hline Diabetes, n (\%) & 455 & $115(25)$ \\
\hline Hypertension, n (\%) & 455 & $291(64)$ \\
\hline Hyperlipidemia, n (\%) & 455 & $160(35)$ \\
\hline Ischemic stroke or transient ischemic attack, n (\%) & 455 & $53(12)$ \\
\hline Cardiovascular disease, $\mathrm{n}(\%)$ & 455 & $57(13)$ \\
\hline Oral anticoagulants, n (\%) & 455 & $35(8)$ \\
\hline \multicolumn{3}{|l|}{ Preoperative symptoms } \\
\hline Total iNPH scale, median (IQR) & 453 & $50(37-62)$ \\
\hline Motor domain, median (IQR) & 451 & $32(18-55)$ \\
\hline Cognitive domain, median (IQR) & 265 & $50(33-67)$ \\
\hline Continence domain, median (IQR) & 439 & $60(40-80)$ \\
\hline Balance domain, median (IQR) & 442 & $67(67-83)$ \\
\hline MMSE, median (IQR) & 446 & $25(21-27)$ \\
\hline Evans'index, median (IQR) & 455 & $0.37(0.34-0.40)$ \\
\hline DESH, n (\%) & 455 & $382(84)$ \\
\hline
\end{tabular}

IQR interquartile range, iNPH idiopathic normal pressure hydrocephalus; MMSE mini-mental state examination, DESH disproportionately enlarged subarachnoid-space hydrocephalus

\section{Imaging markers}

Evans' index and disproportionately enlarged subarachnoid space hydrocephalus (DESH) was assessed on preoperative computed tomography (CT) scans of the brain (CT in 430 patients and MRI in 25 patients). The scans were performed median 1 day (IQR: $1-3$ ) before the shunt surgery. Evans' index and DESH were assessed as previously described [24, 25]. Evans' index was included as a covariate in all regression analyses as a measure of ventricular volume while DESH was analyzed as a separate predictive variable.

\section{Statistical analysis}

The biomarker values were $\log _{2}$-transformed before all analyses to not violate assumption of normality. Also, time to shunt (in months) was log2-transformed after adding a pseudo-count of 1 . To investigate the association between surgery outcome (delta iNPH scale) and biomarker levels, a linear regression analysis was performed, adjusting for age, sex, waiting time for shunt surgery, iNPH-scale score at baseline and Evans' index. Each CSF biomarker and DESH was analyzed separately, with shunt surgery outcome as the dependent variable. The regression coefficient $\beta$ for the biomarker was calculated and assessed using a t-test. As the biomarker level is on the $\log _{2}$ scale, the beta value is the expected increase in delta iNPH scale when the biomarker level is doubled. Multivariate predictive orthogonal projections to latent structures (OPLS) models [26] predicting outcome 12 months after surgery were constructed: (1) based on sets of basic clinical data (base) including: age, sex, waiting time for shunt surgery, iNPH score at baseline and presence of selected comorbidities (diabetes mellitus, hyperlipidemia, hypertension and previous stroke/TIA or myocardial infarction,); (2) basic data with the addition of the imaging markers Evans' index and DESH; (3) basic data with addition of levels of markers in CSF (NfL, T-tau, P-tau and A $31-42$ ), and (4) finally all above listed variables together, using the R-package ropls [27].

To investigate associations between levels of biomarkers and preoperative symptoms at baseline, linear models were fitted, adjusting for age, sex and Evans' index. The significance was assessed using a t-test. Differences in levels of biomarkers between patients with deterioration and patients with improvement were analyzed with the Mann Whitney U-test. Reliability between the two versions of the Stroop tests was tested with an intraclass correlation coefficient. Analyses were performed using $R$ version 4.0.2 and SPSS version 27; $\mathrm{p}<0.05$ was considered significant.

\section{Results}

During the study period, 491 patients (257 male [57\%]) with iNPH were operated with shunt implantation. Levels of at least one CSF marker and evaluation of symptoms at baseline were available in 455 patients, who were included in the statistical analysis. Median age at time of shunt surgery was 75 years (range 50-89). Two hundred forty-five of 376 patients $(65 \%)$ improved $>0$ points on the iNPH scale in analyses of outcome and 205 (55\%) improved $\geq 5$ points (delta iNPH scale $>5$ ). The median preoperative iNPH scale score was 50 [IQR: 37-62] and the median score 12 months after shunt surgery 56 [IQR: 41-73]. Symptoms and comorbidities are presented in Table 1 . The preoperative iNPH scale score was lower (more severe symptoms) in the 79 patients excluded from analyses of outcome compared with the included 376 included patients (44 (IQR: $31-58$ ) vs 50 (IQR: 38-63), $\mathrm{p}=0.016$ ). There was no difference between included and excluded patients regarding other descriptive data.

NfL levels were higher than the reference range in $67(29 \%)$ of all patients and in $25(22 \%)$ of shunt responders (delta iNPH $>5$ points). The proportions of patients with levels of CSF biomarkers outside reference ranges are presented in Table 2. There was a significant negative association between shunt surgery outcome 12 months post-operative (delta iNPH scale) 
Table 2 Median CSF biomarker levels in all patients and proportion of patients with levels outside reference range in the whole sample and in patients with postoperative improvement

\begin{tabular}{lllll}
\hline & $\mathbf{n}$ & Median (IQR) & $\begin{array}{l}\text { Outside reference range, } \mathbf{n} \\
(\%)\end{array}$ & $\begin{array}{l}\text { Outside reference range } \\
\text { among shunt responders } \\
\mathbf{n}(\%)\end{array}$ \\
\hline $\mathrm{NfL}, \mathrm{ng} / \mathrm{L}$ & & & $25(22)$ \\
$\mathrm{T}-\mathrm{tau}, \mathrm{ng} / \mathrm{L}$ & 234 & $1175(850-1970)$ & $67(29)$ & $29(14)$ \\
$\mathrm{P}-\mathrm{tau}, \mathrm{ng} / \mathrm{L}$ & 442 & $200(145-282)$ & $73(17)$ & $12(6)$ \\
$\mathrm{A} \beta 1-42, \mathrm{ng} / \mathrm{L}$ & 436 & $29(23-37)$ & $30(7)$ & $93(45)$ \\
\hline
\end{tabular}

CSF cerebrospinal fluid, NfL neurofilament light protein, T-tau total tau, $P$-tau phosphorylated tau, $A \beta 1-42$ amyloid beta1-42, IQR interquartile range Normal reference ranges: A $\beta 1-42:>530 \mathrm{ng} / \mathrm{L} ; \mathrm{NfL}: 40-60$ years, $<890 \mathrm{ng} / \mathrm{L},>60$ years, $<1850 \mathrm{ng} / \mathrm{L} ; \mathrm{T}$-tau: $<350 \mathrm{ng} / \mathrm{L} ; \mathrm{P}-\mathrm{Tau}:<60 \mathrm{ng} / \mathrm{L}$

${ }^{1}$ Improvement $\geq 5$ points on iNPH scale 12 months after shunt surgery

Table 3 A linear model was built for each predictor relative to the outcome 12 months postoperative change on the iNPH scale (delta iNPH scale) at follow-up and the covariates age, sex, waiting time for shunt surgery, iNPH scale score at baseline and Evans' index

\begin{tabular}{llc}
\hline & Coefficient & $\begin{array}{l}\text { Analysis of } \\
\text { variance } \\
\text { P-value }\end{array}$ \\
\hline NfL $(n=187)$ & -3.10 & $\mathbf{0 . 0 1 6}$ \\
T-tau $(n=364)$ & -2.45 & $\mathbf{0 . 0 1 2}$ \\
P-tau $(n=362)$ & -0.62 & 0.65 \\
Aß1-42 $(n=363)$ & 2.76 & 0.058 \\
DESH $(n=376)$ & 4.30 & 0.055 \\
Covariates: & & \\
Age & -0.50 & $<\mathbf{0 . 0 0 1}$ \\
Sex & -1.39 & 0.42 \\
Time to shunt & -6.03 & $<\mathbf{0 . 0 0 1}$ \\
iNPH scale baseline & -0.23 & $<\mathbf{0 . 0 0 1}$ \\
Evans' index & 20.08 & 0.33 \\
\hline
\end{tabular}

iNPH idiopathic normal pressure hydrocephalus, $N f L$ neurofilament light protein, T-tau total tau, $P$-tau phosphorylated tau, $A \beta 1-42$ amyloid beta-42; DESH disproportionately enlarged subarachnoid-space hydrocephalus

Bold numbers are significant

with higher levels of NfL $(\beta=-3.10, p=0.016)$ and $\mathrm{T}$-tau $(\beta=-2.45, \mathrm{p}=0.012)$. There were no associations between levels of P-tau or A $\beta 1-42$ with outcome after shunt surgery 12 months post operatively. There was a trend, but no significant association, between DESH and outcome after shunt surgery $(\beta=4.30, p=0.055)$, Table 3 . The models were adjusted for age, sex, waiting time for shunt surgery, preoperative iNPH scale score and Evans' index, Table 3. Associations between CSF biomarkers and outcome 3 months after shunt surgery are presented in Additional file 1: Table S2. At 3 months after shunting there was a significant association between shunt surgery outcome with levels of NfL $(\beta=-2.22, p=0.026)$ and $A \beta 1-42(\beta=-3.95$, $\mathrm{p}=0.0013)$. Only 187 patients in the analyses of outcome were investigated with preoperative NfL compared with 364 who were investigated with T-tau. Therefore, the association between CSF biomarkers and outcome after shunt surgery (12 months) for all CSF markers and DESH were also investigated only in the individuals with NfL data. In this analysis the association between $\mathrm{T}$-tau and $\mathrm{A} \beta 1-42$ with outcome were slightly weaker, $\beta=-1.66(p=0.22)$ and $\beta=3.14$ $(\mathrm{p}=0.12)$, respectively, Additional file 1: Table S3.

NfL was higher in patients who deteriorated after shunt surgery 12 months postoperative than in patients with postoperative iNPH scale improvement (1250 [IQR:1020-2220] vs. 1020 [770-1649], p < 0.001). T-tau was higher in patients who deteriorated after shunt surgery than in patients with postoperative iNPH scale improvement (221 [IQR: 159-346] vs. 190 [135-261], $\mathrm{p}=0.0039$ ). There was no significant difference in P-tau or A $\beta 1-42$ between patients who deteriorated and patients who did not, $\mathrm{p}=0.061$ and $\mathrm{p}=0.057$, respectively, Fig. 2.

To investigate if basic clinical data including comorbidities with the addition of imaging markers and CSF markers can be used to improve surgery outcome prediction 12 months after surgery, four OPLS models were trained, stepwise adding variables to the models. At follow-up 12 months after shunt surgery the base model (base data + comorbidities) showed a weak association to surgery outcome, as can be seen in the cross validated score ( $\mathrm{pQ} 2<0.01, \mathrm{Q} 2=0.156)$. The most important variable was waiting time for shunt surgery $(\mathrm{VIP}=2.08)$, Table 4 and Additional file 1: Fig. S1. Adding additional variables to the model did not improve performance in predicting surgery outcome, as can be seen by no major improvements in $\mathrm{pQ} 2$ was achieved. A very weak improvement was found adding imaging markers $(\mathrm{pQ} 2<0.01, \mathrm{Q}=0$. 16) compared with only base data (Additional file 1 : Fig. S2), but adding CSF markers did not improve the 


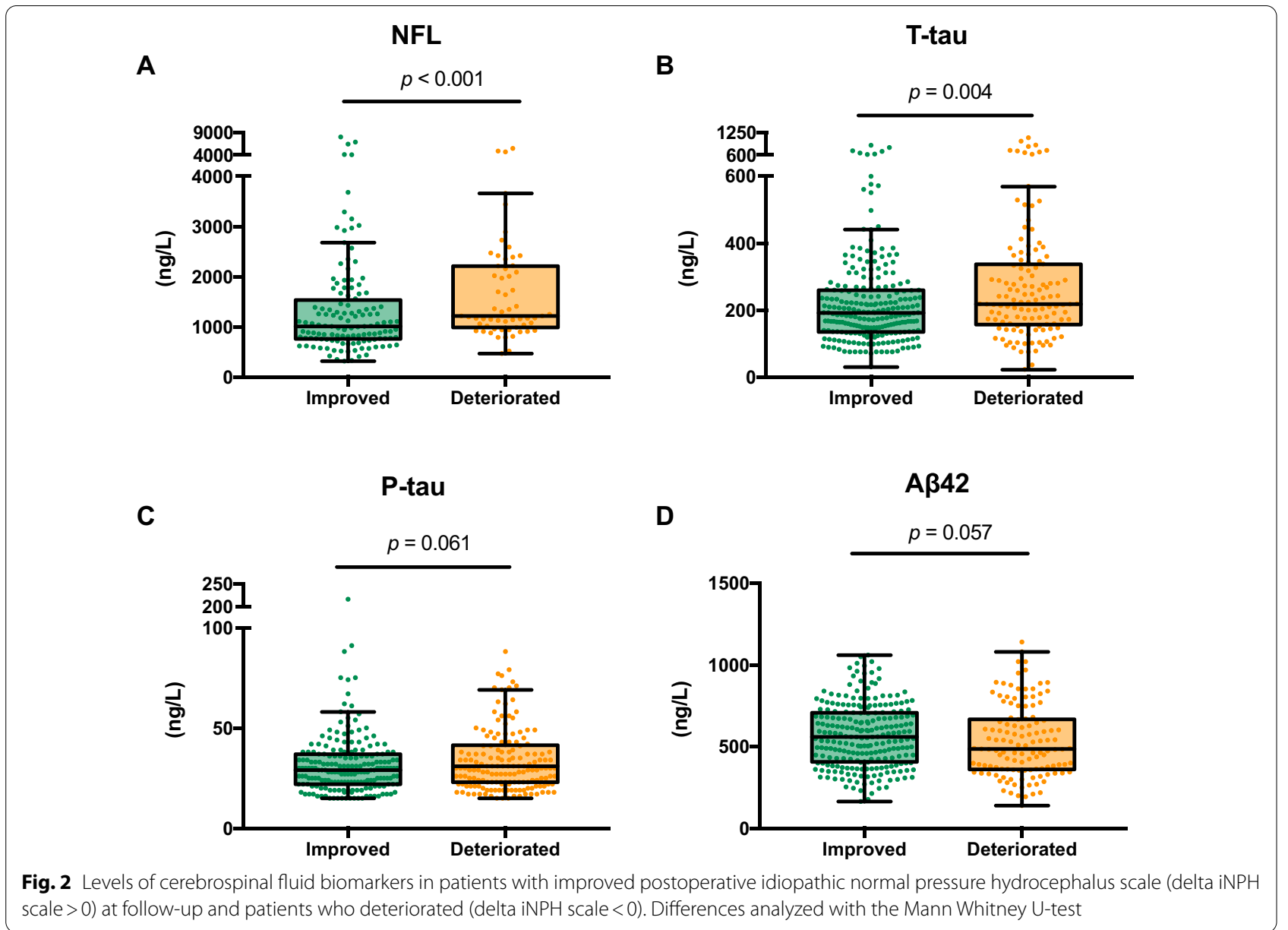

Table 4 Four OPLS predicting outcome 12 months after shunt surgery

\begin{tabular}{lllll}
\hline Variables: & R2Y & Q2 & pR2Y & pQ2 \\
\hline 1. Base & 0.192 & 0.156 & 0.01 & 0.01 \\
2. Base+Evans' index+DESH & 0.203 & 0.16 & 0.01 & 0.01 \\
3. Base+CSF markers & 0.22 & 0.151 & 0.01 & 0.01 \\
4. Base+Evans + DESH + CSF markers & 0.231 & 0.157 & 0.01 & 0.01 \\
\hline
\end{tabular}

The first model (1) based on basic clinical data (base) including: age, sex, waiting time for shunt surgery, iNPH score at baseline and presence the comorbidities: diabetes mellitus, hyperlipidemia, hypertension and previous stroke or myocardial infarction; (2) basic data with the addition of the imaging markers Evans' index and DESH; (3) basic data with addition of levels of markers in CSF (NfL, T-tau, P-tau and A 1 1-42), and (4) finally all above listed variables together iNPH idiopathic normal pressure hydrocephalus, NfL neurofilament light protein, $T$-tau total tau, $P$-tau phosphorylated tau, AB1-42 amyloid beta-42, DESH disproportionately enlarged subarachnoid-space hydrocephalus

model ( $\mathrm{pQ} 2<0.01, \mathrm{Q}=0.151)$, Additional file 1: Fig. S3. In all models, age, waiting time to shunt surgery and iNPH score at baseline were the most important variables associated with outcome after shunt surgery (highest
VIP). Figure 3 illustrates the model with all variables included.

There were associations between higher NfL and lower total score on the iNPH scale at baseline. There was an association between higher $A \beta 1-42$ and better cognitive function at baseline, whether this was measured with MMSE or the cognitive domain of the iNPH scale. All associations between symptoms at baseline and CSF markers are presented in Table 5 .

The reliability, tested with intraclass correlation coefficient between the original Stroop tests and the alternative versions with only 24 colored circles/words, was 0.89 for Stroop color and 0.73 for Stroop interference.

\section{Discussion}

This is, to our knowledge, the largest study assessing the association between preoperative CSF biomarkers with outcome after shunt surgery in patients with iNPH. In this single-center study of 455 patients with iNPH, of whom 376 were examined before and 12 months after shunt surgery, levels of CSF biomarkers were compared with outcome after shunt surgery and symptoms 


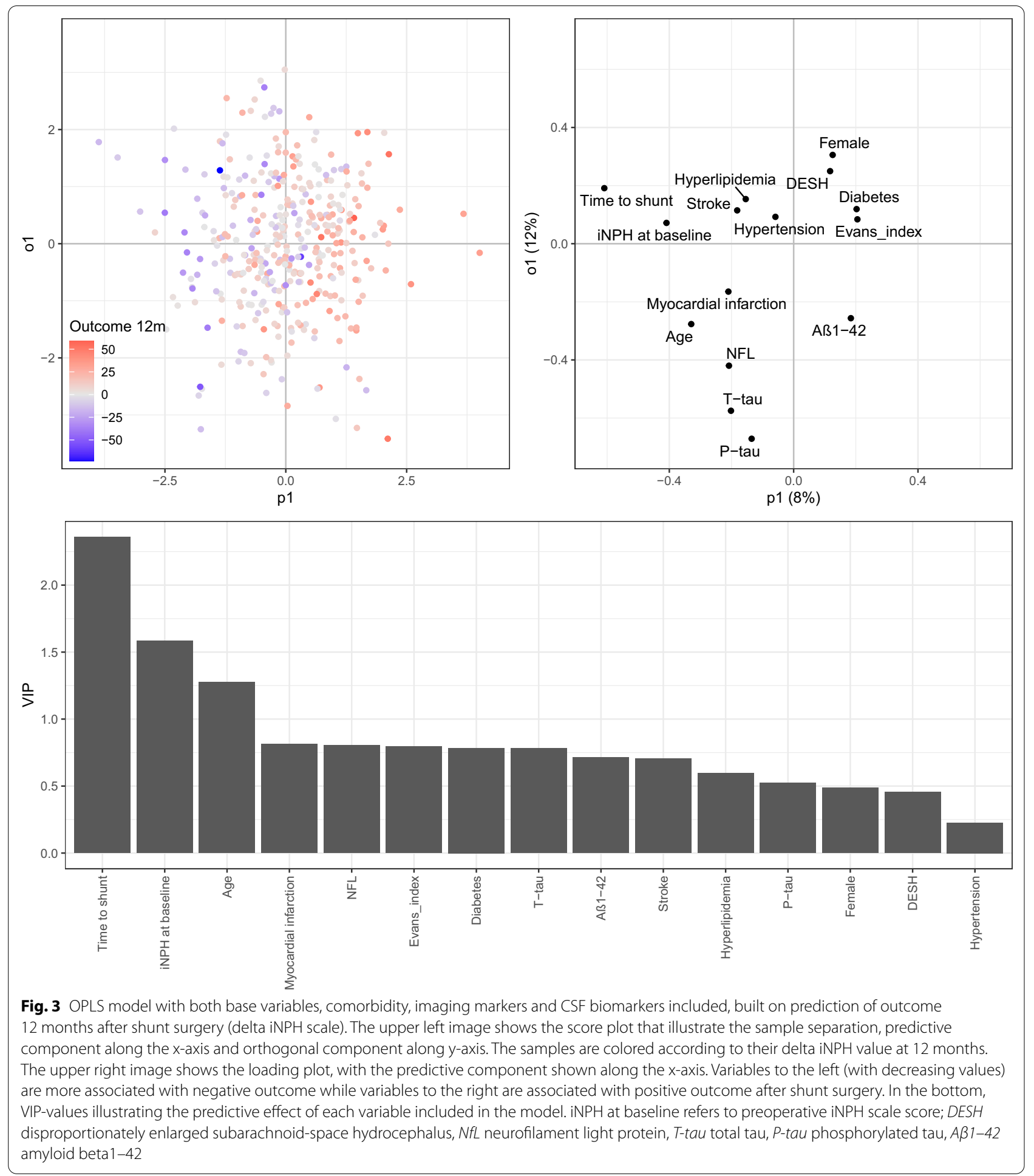

at baseline. Higher preoperative levels of NfL and T-tau in CSF were associated with less improvement one year after shunt surgery. Patients who deteriorated after surgery had higher preoperative levels of NfL and T-tau. However, half of the patients who improved after shunt surgery had preoperative levels of $A \beta 1-42$ below reference ranges, and $\mathrm{NfL}$ was above reference range in $22 \%$ of the shunt responders. Combining CSF biomarkers with imaging data and data of comorbidity did not improve the ability to predict outcome after shunt surgery. This 
Table 5 Coefficients from a linear regression model for each CSF marker with preoperative symptoms at baseline as outcome and the covariates age, sex and Evan's index

\begin{tabular}{|c|c|c|c|c|c|c|}
\hline CSF marker & Total iNPH scale & Motor domain & Cognitive domain & Balance domain & Continence domain & MMSE \\
\hline NfL & $-4.24 * * *$ & $-5.23^{* * *}$ & -1.80 & $-3.73^{*}$ & $-4.65^{* *}$ & -0.66 \\
\hline T-tau & -0.24 & 0.50 & 1.63 & -0.15 & -1.54 & -0.32 \\
\hline P-tau & $3.26^{*}$ & $4.57^{*}$ & 3.42 & -0.78 & $4.31^{*}$ & -0.22 \\
\hline$A \beta 1-42$ & 0.86 & -0.32 & $8.24^{* * *}$ & 1.42 & 0.73 & $1.62^{* * *}$ \\
\hline
\end{tabular}

CSF cerebrospinal fluid, iNPH idiopathic normal pressure hydrocephalus, NfL neurofilament light protein, $T$-tau total tau, $P$-tau phosphorylated tau, $A \beta 1-42$ amyloid beta1-42

Bold numbers are significant. ${ }^{*}<0.05 ;{ }^{* *}<0.01 ;{ }^{* * *}<0.001$

indicates that the CSF markers included here are not suitable for excluding patients from shunt surgery.

\section{Predictive value of CSF biomarkers}

This study indicated an association between higher preoperative levels of $\mathrm{NfL}$ and poorer outcome measured with the iNPH scale. This is in line with a study by Tullberg et al. reporting that NfL may be a clinically useful biomarker for good prognosis in iNPH [28].

High NfL levels have previously been described in patients with $\mathrm{NPH}$, but also frontotemporal dementia, $\mathrm{AD}$ and vascular dementia [29]. The association between high NfL and worse outcome in iNPH could therefore be explained by comorbidity with other neurodegenerative disorders in some patients. Cerebrovascular disease is common in iNPH and it is possible that concomitant vascular disease is the explanation for both increased NfL and its association with less improvement after shunt surgery $[29,30]$.

There was also an association between higher preoperative levels of $\mathrm{T}$-tau and a less favorable outcome one year after shunt. There was no association between P-tau and outcome after shunting. This is consistent with most previous studies, which have reported no difference in P-Tau between patients who deteriorated and patients who did not [12]. However, one study reported that levels of P-tau were associated with cognitive outcome after shunting [31] In line with our results, increased T-tau has been suggested to predict less postoperative improvement in gait function [18]. The levels of T-tau and P-tau have previously been compared between $\mathrm{AD}$ and $\mathrm{iNPH}$, and found to be higher in AD than in iNPH [18]. Furthermore, T-tau is lower in iNPH than in healthy elderly subjects, at a group level [32]. It therefore seems possible that individuals with increased T-tau and P-tau with iNPH might suffer from neurodegenerative comorbidity. This could be explained by the tau-pathogenesis hypothesis [33]. T-tau and P-tau are microtubule-associated proteins found in the cytoskeleton of mainly non-myelinated axons in the CNS. This structure is seen in cortical areas of the brain, especially in regions involved in memory function, such as the limbic cortex, including the hippocampus [33, 34].

The association between $A \beta 1-42$ and shunt surgery outcome was not as strong as those for NfL and T-tau. A previous study reported that $A \beta 1-42$ could predict outcome at 6-month follow-up and that a combination of A $\beta 1-42$ and T-tau could predict outcome after shunting. Differences compared with our study were a relatively small sample size and that their samples were collected from the ventricles [17]. Levels of $A \beta 1-42$ are decreased in $\mathrm{AD}$, but in previous studies have also been shown to be lower in iNPH than in healthy controls $[18,32]$. It has therefore been questioned whether $A \beta 1-42$ could aid discrimination between iNPH and $\mathrm{AD}[15,18,32]$. One study also links low levels of $A \beta 1-42$ to a more definitive cognitive impairment that could suggest $A D$ pathology [35]. In $A D$, there is a selective reduction of $A \beta 1-42 \mathrm{com}-$ pared with the shorter and more hydrophilic $A \beta 1-38$ and $A \beta 1-40$ peptides in CSF [36]. This is due to sequestration of $A \beta 1-42$ in amyloid plaques in the brain. In iNPH, on the other hand, there is a concordant reduction in $A \beta 1$ 38, $A \beta 1-40$ and $A \beta 1-42$ [36]. The molecular mechanism is unknown, but the levels normalize following successful shunt surgery [36]. Hence, CSF $A \beta 1-42 / A \beta 1-40$ is a better marker for $A \beta$ plaque pathology in conditions in which disturbed CSF dynamics can be expected. Unfortunately, we did not have access to CSF $A \beta 1-38$ or $A \beta 1$ 40 measures in the current study.

\section{Associations between CSF biomarkers and preoperative symptoms}

Higher NfL was associated with lower scores on the total iNPH scale as well as on the separate motor, balance and continence domains (Table 5). Possible explanations include white matter injuries from compressing enlarged ventricles, or trans-ependymal CSF passage with excessive white matter edema or concomitant cerebrovascular disease with white matter lesions [28, 30]. In line with our results, previous studies have found that preoperative 
NfL levels correlate with the severity of clinical symptoms $[8,12,28]$. We found an association, after controlling for age and sex, between lower $A \beta 1-42$ and cognitive impairment, measured with either MMSE or the cognitive domain from the iNPH scale. This could be related to comorbidity with neurodegenerative disease such as AD, as mentioned above, and is in congruence with previous studies of both CSF and cortical biopsies [37-39].

\section{Outcome in patients with pathological levels of CSF markers}

Although there were associations between a less favorable outcome and high levels of NfL and T-tau, a majority of the patients with pathologically altered levels of the CSF biomarkers responded well to shunt surgery. To investigate if basic clinical data, including comorbidities, imaging markers and CSF markers can be used to improve surgery outcome prediction we stepwise trained a set of OPLS models. In all models, age, waiting time to shunt surgery and iNPH score at baseline were the most important variables associated with outcome after shunt surgery. This strengthens our suggestion that the now investigated CSF biomarkers may have differential diagnostic value, but patients should not be excluded from shunt surgery because of abnormal CSF biomarker levels.

Some limitations should be considered. There were long waiting times for shunt surgery at our center during the study period, due to insufficient capacity. Long waiting times negatively affect the outcome after shunt surgery $[40,41]$ and patients deteriorate while waiting for surgery. No new clinical assessments were performed right before shunt surgery. This is the probable cause for the lower proportion of patients who improved in this study (55\%) compared with in previous literature. This also explains why waiting time for surgery was the variable with strongest association with outcome. Furthermore, outcome after shunting is most favorable when the time from onset of symptoms to surgery is minimized [42]. In the present material, time from onset of symptoms to examination was estimated to be $1-8$ years but was not included in the statistical analysis, since this variable can be uncertain and requires reliable information from a relative. The follow-up at 12 months after shunt surgery is late compared with in many studies in the field, but an even longer follow-up time might have revealed more concomitant neurodegenerative disorders. Unfortunately, we did not have data that extended longer than 12 months. However, the purpose of this study was to investigate associations between levels of biomarkers with outcome of shunt surgery and we believe that 12 months follow-up is sufficient to assess the result of shunt surgery.
We did not have data of years of education, that may be useful data when interpreting results of cognitive testing. In a retrospective study such as this one, there is always a risk of inclusion bias since some patients may have been excluded from shunt surgery (and therefore this study) based on highly pathological levels of the CSF biomarkers. Furthermore, patients excluded from the outcome analyses had more severe preoperative symptoms.

Two radiological measures were included. However, white matter changes that are believed to represent cerebrovascular changes have been associated with deterioration after surgery [43]. The association between high $\mathrm{NfL}$ and less favorable outcome could also be attributed to more pronounced white matter brain lesions.

\section{Conclusions}

Higher levels of NfL and T-tau are associated with a less favorable response to shunt surgery. High levels of NfL were associated with more pronounced motor symptoms before surgery and lower levels of $A \beta 1-42$ were associated with more cognitive dysfunction. These associations may be explained by concomitant subcortical vascular injuries and $\mathrm{AD}$ pathology, respectively. However, the majority of patients with increased CSF levels of NfL and T-tau responded to shunt surgery and inclusion of CSF biomarkers, imaging markers and comorbidity in multivariate predictive OPLS models did not improve predictability in outcome after surgery. We conclude that none of these CSF biomarkers should be used to exclude patients from surgery.

\section{Abbreviations}

iNPH: Idiopathic normal pressure hydrocephalus; CSF: Cerebrospinal fluid; NfL: Neurofilament light chain protein; T-tau: Total tau; P-tau: Phosphorylated tau; Aß1-42: Amyloid beta1-42; IQR: Interquartile range; AD: Alzheimer's disease; MMSE: Mini Mental State Examination; CNS: Central nervous system; DESH: Disproportionately enlarged subarachnoid space hydrocephalus.

\section{Supplementary Information}

The online version contains supplementary material available at https://doi.
org/10.1186/s12987-022-00306-2.

Additional file 1: Table S1. Conversion table for the alternative versions of Stroop color and Stroop interference with only 24 colored circles/ words. Table S2. A linear model was built for each predictor relative to the outcome 3 month postoperative change on the iNPH scale (delta iNPH scale 3 months) at follow-up and the covariates age, sex, waiting time for shunt surgery, iNPH scale score at baseline and Evans' index. Table S3. Including only patients with preoperative NfL. A linear model was built for each predictor relative to the outcome 12-month postoperative change on the iNPH scale (delta iNPH scale) at follow-up and the covariates age, sex, waiting time for shunt surgery, iNPH scale score at baseline and Evans' index. Figure S1. OPLS models built on prediction of outcome 12 months after shunt surgery (delta iNPH scale). The first model (Supplementary figure 1) based on basic clinical data (base) including: age, sex, waiting time for shunt surgery, iNPH score at baseline and presence of the comorbidities: diabetes mellitus, hyperlipidemia, hypertension and previous stroke 
or myocardial infarction. Figure $\mathbf{S 2}$. includes basic data with the addition of the imaging markers Evans' index and DESH. Figure S3. includes basic data with addition of levels of markers in CSF (NfL, T-tau, P-tau and A 1 1-42). The model with all above variables included are included in the main manuscript (Figure 3). The upper left image shows the score plot that illustrate the sample separation, predictive component along the $x$-axis and orthogonal component along $y$-axis. The samples are colored according to their delta iNPH value at 12 months. The upper right image shows the loading plot, with the predictive component shown along the $x$-axis. Variables to the left (with decreasing values) are more associated with negative outcome while variables to the right are associated with positive outcome after shunt surgery. In the bottom, VIP-values illustrating the predictive effect of each variable included in the model. iNPH at baseline refers to preoperative iNPH scale score; DESH = disproportionately enlarged subarachnoid-space hydrocephalus; $\mathrm{NfL}=$ neurofilament light protein; T-tau $=$ total tau; P-tau $=$ phosphorylated tau; $A \beta 1-42=$ amyloid beta1-42.

\section{Authors' contributions}

All authors reviewed, read and approved the final manuscript. MB, JV, DN and VN drafted the manuscript, MB collected the data for the study, JV designed the study, CB and KK performed statistical analyses, $\mathrm{HZ}$ and $\mathrm{KB}$ supervised and interpreted laboratory analyses.

\section{Funding}

JV is supported by the Swedish Society for Medical Research. DN, VN and MB are supported by Region Uppsala ALF-grants. KK is supported by Region Uppsala (ALF-grant and R\&D funds), Uppsala University (R\&D funds), Gun and Bertil Stohne's Foundation, Geriatriska Fonden and Stiftelsen för Gamla Tjänarinnor. $\mathrm{HZ}$ is a Wallenberg Scholar supported by grants from the Swedish Research Council (\#2018-02532), the European Research Council (\#681712), Swedish State Support for Clinical Research (\#ALFGBG-720931), the Alzheimer Drug Discovery Foundation (ADDF), USA (\#201809-2016862), the AD Strategic Fund and the Alzheimer's Association (\#ADSF-21-831376-C, \#ADSF-21-831381-C and \#ADSF-21-831377-C), the Olav Thon Foundation, the Erling-Persson Family Foundation, Stiftelsen för Gamla Tjänarinnor, Hjärnfonden, Sweden (\#FO20190228), the European Union's Horizon 2020 research and innovation program under the Marie Skłodowska-Curie grant agreement No 860197 (MIRIADE) and the UK Dementia Research Institute at UCL.

KB is supported by the Swedish Research Council (\#2017-00915), the Alzheimer Drug Discovery Foundation (ADDF), USA (\#RDAPB-2018092016615), the Swedish Alzheimer Foundation (\#AF-742881), Hjärnfonden, Sweden (\#FO2017-0243), the Swedish state through the agreement between the Swedish government and the County Councils, the ALF agreement (\#ALFGBG-715986), the European Union Joint Program for Neurodegenerative Disorders (JPND2019-466-236) and the National Institute of Health (NIH), USA, (grant \#1R01AG068398-01). Open access funding provided by Uppsala University.

\section{Availability of data and materials}

The datasets analyzed during the current study are available from the corresponding author on reasonable request.

\section{Declarations}

\section{Ethics approval and consent to participate}

The study was approved by the Swedish National Ethical Review Authority (Dnr 2015/174/3 and 2019-06566).

\section{Competing interests}

$\mathrm{HZ}$ has served at scientific advisory boards for Alector, Eisai, Denali, Roche Diagnostics, Wave, Samumed, Siemens Healthineers, Pinteon Therapeutics, Nervgen, AZTherapies and CogRx, has given lectures at symposia sponsored by Cellectricon, Fujirebio, Alzecure and Biogen, and is a co-founder of Brain Biomarker Solutions in Gothenburg AB, which is a part of the GU Ventures Incubator Program, all unrelated to the work presented in this paper. $\mathrm{KB}$ has served as a consultant, on advisory boards, or in data monitoring committees for Abcam, Axon, Biogen, JOMDD/Shimadzu. Julius Clinical, Lilly,
MagQu, Novartis, Prothena, Roche Diagnostics, and Siemens Healthineers, and is a co-founder of Brain Biomarker Solutions in Gothenburg AB, which is a part of the GU Ventures Incubator Program, all unrelated to the work presented in this paper.

\section{Author details}

${ }^{1}$ Department of Neuroscience, Neurology, Uppsala University, Akademiska sjukhuset, ing 85, 75185 Uppsala, Sweden. ' 2 Department of Medical Sciences, Clinical Chemistry, Uppsala University, Uppsala, Sweden. ${ }^{3}$ Department of Surgical Sciences, Radiology, Uppsala University, Uppsala, Sweden. ${ }^{4}$ Department of Cell and Molecular Biology, Uppsala University, Uppsala, Sweden. ${ }^{5}$ Department of Psychiatry and Neurochemistry, Institute of Neuroscience and Physiology, the Sahlgrenska Academy at the University of Gothenburg, Mölndal, Sweden. ${ }^{6}$ Clinical Neurochemistry Laboratory, Sahlgrenska University Hospital, Mölndal, Sweden. ${ }^{7}$ Department of Neurodegenerative Disease, UCL Institute of Neurology, Queen Square, London, UK. ${ }^{8}$ UK Dementia Research Institute at UCL, London, UK. ${ }^{9}$ Hong Kong Center for Neurodegenerative Diseases, Hong Kong, China.

\section{Received: 23 September 2021 Accepted: 15 January 2022} Published: 14 February 2022

\section{References}

1. Jaraj D, Rabiei K, Marlow T, Jensen C, Skoog I, Wikkelsø C. Prevalence of idiopathic normal-pressure hydrocephalus. Neurology. 2014;82(16):1449-54.

2. Brean A, Eide PK. Prevalence of probable idiopathic normal pressure hydrocephalus in a Norwegian population. Acta Neurol Scand. 2008;118(1):48-53.

3. Sundström N, Malm J, Laurell K, Lundin F, Kahlon B, Cesarini KG, et al. Incidence and outcome of surgery for adult hydrocephalus patients in Sweden. Br J Neurosurg. 2017;31(1):21-7.

4. Toma AK, Papadopoulos MC, Stapleton S, Kitchen ND, Watkins LD. Systematic review of the outcome of shunt surgery in idiopathic normalpressure hydrocephalus. Acta Neurochir. 2013;155(10):1977-80.

5. Klinge P, Hellström P, Tans J, Wikkelsø C, European iNPH Multicentre Study Group. One-year outcome in the European multicentre study on $\mathrm{iNPH}$. Acta Neurol Scand. 2012;126(3):145-53.

6. Graff-Radford NR. Alzheimer CSF biomarkers may be misleading in normal-pressure hydrocephalus. Neurology. 2014;83(17):1573-5.

7. Gaetani L, Blennow K, Calabresi P, Di Filippo M, Parnetti L, Zetterberg H. Neurofilament light chain as a biomarker in neurological disorders. J Neurol Neurosurg Psychiatry. 2019;90(8):870-81.

8. Tullberg M, Blennow K, Månsson J-E, Fredman P, Tisell M, Wikkelsö C Cerebrospinal fluid markers before and after shunting in patients with secondary and idiopathic normal pressure hydrocephalus. Fluids Barriers CNS. 2008;5(1):9.

9. Tullberg M, Blennow K, Månsson J-E, Fredman P, Tisell M, Wikkelsö C Ventricular cerebrospinal fluid neurofilament protein levels decrease in parallel with white matter pathology after shunt surgery in normal pressure hydrocephalus. Eur J Neurol. 2007;14(3):248-54.

10. Craven CL, Baudracco I, Zetterberg H, Lunn MPT, Chapman MD, Lakdawala N, et al. The predictive value of T-tau and AB1-42 levels in idiopathic normal pressure hydrocephalus. Acta Neurochir (Wien). 2017:159(12):2293-300

11. Jeppsson A, Wikkelsö C, Blennow K, Zetterberg H, Constantinescu R, Remes AM, et al. CSF biomarkers distinguish idiopathic normal pressure hydrocephalus from its mimics. J Neurol Neurosurg Psychiatry. 2019;90(10):1117-23.

12. Agren-Wilsson A, Lekman A, Sjöberg W, Rosengren L, Blennow K, Bergenheim AT, et al. CSF biomarkers in the evaluation of idiopathic normal pressure hydrocephalus. Acta Neurol Scand. 2007;1 16(5):333-9.

13. Kapaki EN, Paraskevas GP, Tzerakis NG, Sfagos C, Seretis A, Kararizou E, et al. Cerebrospinal fluid tau, phospho-tau181 and beta-amyloid 1-42 in idiopathic normal pressure hydrocephalus: a discrimination from Alzheimer's disease. Eur J Neurol. 2007;14(2):168-73.

14. Ray B, Reyes PF, Lahiri DK. Biochemical studies in Normal Pressure Hydrocephalus (NPH) patients: change in CSF levels of amyloid precursor 
protein (APP), amyloid-beta (A $\beta$ ) peptide and phospho-tau. J Psychiatr Res. 2011;45(4):539-47.

15. Miyajima M, Nakajima M, Ogino I, Miyata H, Motoi Y, Arai H. Soluble amyloid precursor protein a in the cerebrospinal fluid as a diagnostic and prognostic biomarker for idiopathic normal pressure hydrocephalus. Eur J Neurol. 2013;20(2):236-42.

16. Kang K, Ko P-W, Jin M, Suk K, Lee H-W. Idiopathic normal-pressure hydrocephalus, cerebrospinal fluid biomarkers, and the cerebrospinal fluid tap test. J Clin Neurosci. 2014:21(8):1398-403.

17. Tarnaris A, Toma Ahmed K, Chapman MD, Keir G, Kitchen ND, Watkins LD. Use of cerebrospinal fluid amyloid- $\beta$ and total tau protein to predict favorable surgical outcomes in patients with idiopathic normal pressure hydrocephalus: clinical article. JNS. 2011;115(1):145-50.

18. Jingami N, Asada-Utsugi M, Uemura K, Noto R, Takahashi M, Ozaki A, et al. Idiopathic normal pressure hydrocephalus has a different cerebrospinal fluid biomarker profile from Alzheimer's disease. J Alzheimers Dis. 2015;45(1):109-15.

19. Relkin N, Marmarou A, Klinge P, Bergsneider M, Black PM. Diagnosing Idiopathic Normal-Pressure Hydrocephalus. Neurosurgery. 2005;57(suppl_3):S2-4-S2-16.

20. Hellström P, Klinge P, Tans J, Wikkelsø C. A new scale for assessment of severity and outcome in iNPH. Acta Neurol Scand. 2012;126(4):229-37.

21. Palmqvist $\mathrm{S}$, Zetterberg $\mathrm{H}$, Blennow K, Vestberg $\mathrm{S}$, Andreasson U, Brooks DJ, Owenius R, et al. Accuracy of brain amyloid detection in clinical practice using cerebrospinal fluid $\beta$-amyloid 42: a cross-validation study against amyloid positron emission tomography. JAMA Neurol. 2014:71(10):1282-9.

22. Yilmaz A, Blennow K, Hagberg L, Nilsson S, Price RW, Schouten J, Spudich S, Underwood J, Zetterberg H, Gisslén M. Neurofilament light chain protein as a marker of neuronal injury: review of its use in HIV-1 infection and reference values for HIV-negative controls. Expert Rev Mol Diagn. 2017;17(8):761-70.

23. Hansson O, Zetterberg H, Buchhave P, Londos E, Blennow K, Minthon L. Association between CSF biomarkers and incipient Alzheimer's disease in patients with mild cognitive impairment: a follow-up study. Lancet Neurol. 2006;5(3):228-34.

24. Fällmar D, Andersson O, Kilander L, Löwenmark M, Nyholm D, Virhammar J. Imaging features associated with idiopathic normal pressure hydrocephalus have high specificity even when comparing with vascular dementia and atypical parkinsonism. Fluids Barriers CNS. 2021:18(1):35.

25. Kockum K, Lilja-Lund O, Larsson E-M, Rosell M, Söderström L, Virhammar J, Laurell K. The idiopathic normal-pressure hydrocephalus radscale: a radiological scale for structured evaluation. Eur J Neurol. 2018;25(3):569-76.

26. Trygg J, Wold S. Orthogonal projections to latent structures (O-PLS). J Chemom. 2002;16(3):119-28.

27. Thévenot EA, Roux A, Xu Y, Ezan E, Junot $C$. Analysis of the human adult urinary metabolome variations with age, body mass index, and gender by implementing a comprehensive workflow for univariate and OPLS statistical analyses. J Proteome Res. 2015;14(8):3322-35.

28. Tullberg M, Rosengren L, Blomsterwall E, Karlsson J-E, Wikkelso C. CSF neurofilament and glial fibrillary acidic protein in normal pressure hydrocephalus. Neurology. 1998;50(4):1122-7.

29. Sjögren M, Rosengren L, Minthon L, Davidsson P, Blennow K, Wallin A. Cytoskeleton proteins in CSF distinguish frontotemporal dementia from AD. Neurology. 2000;54(10):1960-4.

30. Sjögren M, Blomberg M, Jonsson M, Wahlund LO, Edman A, Lind K, et al. Neurofilament protein in cerebrospinal fluid: a marker of white matter changes. J Neurosci Res. 2001;66(3):510-6.

31. Nakajima M, Miyajima M, Ogino I, Akiba C, Kawamura K, Kamohara C, et al. Preoperative phosphorylated tau concentration in the cerebrospinal fluid can predict cognitive function three years after shunt surgery in patients with idiopathic normal pressure hydrocephalus. JAD. 2018;66(1):319-31.

32. Schirinzi T, Sancesario GM, lalongo C, Imbriani P, Madeo G, Toniolo S, et al. A clinical and biochemical analysis in the differential diagnosis of idiopathic normal pressure hydrocephalus. Front Neurol. 2015;6:86.

33. Kolarova M, García-Sierra F, Bartos A, Ricny J, Ripova D. Structure and pathology of tau protein in Alzheimer disease. Int J Alzheimers Dis. 2012;2012:1-13.
34. Hu W, Wu F, Zhang Y, Gong C-X, Iqbal K, Liu F. Expression of tau pathology-related proteins in different brain regions: a molecular basis of tau pathogenesis. Front Aging Neurosci. 2017;27(9):311.

35. Lim TS, Choi JY, Park SA, Youn YC, Lee HY, Kim BG, et al. Evaluation of coexistence of Alzheimer's disease in idiopathic normal pressure hydrocephalus using ELISA analyses for CSF biomarkers. BMC Neurol. 2014;14(1):66.

36. Hansson O, Lehmann S, Otto M, Zetterberg H, Lewczuk P. Advantages and disadvantages of the use of the CSF amyloid $\beta$ (AB) $42 / 40$ ratio in the diagnosis of Alzheimer's disease. Alzheimers Res Ther. 2019;11(1):34.

37. Hamilton R, Patel S, Lee EB, Jackson EM, Lopinto J, Arnold SE, et al. Lack of shunt response in suspected idiopathic normal pressure hydrocephalus with Alzheimer disease pathology. Ann Neurol. 2010;68(4):535-40.

38. Leinonen V, Koivisto AM, Alafuzoff I, Pyykkö OT, Rummukainen J, von und zu Fraunberg $\mathrm{M}$, et al. Cortical brain biopsy in long-term prognostication of 468 patients with possible normal pressure hydrocephalus. Neurodegenerative Dis. 2012;10(14):166-9.

39. Cabral D, Beach TG, Vedders L, Sue LI, Jacobson S, Myers K, et al. Frequency of Alzheimer's disease pathology at autopsy in patients with clinical normal pressure hydrocephalus. Alzheimers Dement. 2011;7(5):509-13.

40. Bådagård H, Braun M, Nilsson D, Stridh L, Virhammar J. Negative predictors of shunt surgery outcome in normal pressure hydrocephalus. Acta Neurol Scand. 2020;141(3):219-25.

41. Andren K, Wikkelso C, Tisell M, Hellstrom P. Natural course of idiopathic normal pressure hydrocephalus. J Neurol Neurosurgery Psychiatry. 2014;85(7):806-10.

42. Takeuchi T, Kasahara E, Iwasaki M. Clinical characteristics and indications for shunting in patients with idiopathic normal pressure hydrocephalus with brain atrophy (atypical idiopathic normal pressure hydrocephalus). No Shinkei Geka. 2000;28(6):505-15.

43. Bech-Azeddine R, Hogh P, Juhler M, Gjerris F, Waldemar G. Idiopathic normal-pressure hydrocephalus: clinical comorbidity correlated with cerebral biopsy findings and outcome of cerebrospinal fluid shunting. $J$ Neurol Neurosurgery Psychiatry. 2007;78(2):157-61.

\section{Publisher's Note}

Springer Nature remains neutral with regard to jurisdictional claims in published maps and institutional affiliations.

Ready to submit your research? Choose BMC and benefit from

- fast, convenient online submission

- thorough peer review by experienced researchers in your field

- rapid publication on acceptance

- support for research data, including large and complex data types

- gold Open Access which fosters wider collaboration and increased citations

- maximum visibility for your research: over $100 \mathrm{M}$ website views per year

At BMC, research is always in progress.

Learn more biomedcentral.com/submissions 